Aus dem Garnisonlazarett Erfurt.

\title{
Über die Deckung von Trachealdefekten vermittelst autoplastischer freier Fascientransplantation.
}

\author{
Von Stabsarzt Dr. Münnich.
}

Zur Deckung von Trachealdefekten, die im AnschluB an operative Eingriffe oder im Gefolge von Verletzungen der Trachea (Hieb, Stich, Schuß) sich entwickelt haben, sind bis in die Neuzeit eine größere Anzahl von Operationsmethoden veröffentlicht worden. Die älteren Methoden der einfachen Lappenplastik nach Dieffenbach, Velpeau, Barracz u. a. kommen heutigen Tages bei Deckung größerer Defekte nur noch selten zur Anwendung, da ihr Erfolg unsicher ist und sie außerdem den Anforderungen, die die moderne Chirurgie an eine zuverlässige Trachealplastik stellt, nicht mehr genügen. Denn bei der ausgesprochenen Elastizität der Haut besteht bei allen diesen Verfahren die Gefahr, daß sich die Haut bei tiefen Inspirationen ventilartig in das Lumen der Trachea einstülpt und dadurch zu Stenosen Veranlassung gibt. Des weiteren ist auch mehrfach bei Defekten im Bereich der vorderen und seitlichen Trachealwand ein Hineinwuchern von Granulationsgewebe in die Trachea beobachtet worden, so daß, um einer zunehmenden Stenose vorzubeugen, die Tracheotomie notwendig wurde. Diese genannten Fehler vermeidet am sichersten unter den neueren Methoden die Küster . sche, die Resektion der Trachea. Nur empfiehlt es sich, anstatt des ursprünglich von $\mathrm{Küster}$ angegebenen ringförmigen $\mathrm{Re}$ sektionsschnittes, wenn möglich, einen bajonettförmigen zu verwenden, da auf diese Weise der Entwicklung eines diaphragmaartigen Narbensegels, das sich an der Resektionsstelle bilden könnte, am ehesten vorgebeugt wird. Jedoch sind auch dieser Methode infolge der Schwierigkeit der Adaptierung der Trachealwundränder bei größeren Defekten gewisse Grenzen gesetzt. 
Experimentelle Untersuchüngen an der Leiche durch Nowakowsky haben nämlich ergeben, daß sich Defekte bis $4 \mathrm{~cm}$ gut vereinigen lassen, vorausgesetzt, daß die Halswirbelsäule weder nach vorn noch nach hinten gekrümmt ist. Bei einer Entfeŕnung von $5 \mathrm{~cm}$ stößt jedoch die Vereinigung vielfach bereits auf Schwierigkeiten. Auch in denjenigen Fällen, wo infolge einer vorher angelegten Laryngofissur oder Defekten im Ringknorpel die obere Partie wesentlich anders gestaltet ist wie die röhrenförmige Trachea, ist die Vereinigung nach dieser Methode nicht durchführbar. Gute Resultate, allerdings nur bei kleineren Defekten anwendbar, ergibt auch die Königsche Methode, der zum Verschluß einen gestielten Hautknorpellappen aus dem Schildknorpel entnahm. Das Verfahren ist nicht schwierig, der kosmetische Erfolg befriedigend. v. Mangold überbrückte den Defekt vermittelst eines seitlich von diesem gelegenen Hautlappens, unter den vorher ein Stück Rippenknorpel implantiert war. Ein zweiter, gleichfalls seitlich gewählter Hautlappen wurde in den Defekt gelegt und mit dem umgeschlagenen Hautrippenknorpellappen vereinigt. Auch auf heteroplastischem Wege (Große) ist es bei kleineren Defekten und Erhaltung der seitlichen Trachealwand gelungen, diesen zu decken, und zwar vermittelst eines Silberdrahtnetzes, das zwischen den Defekt. rändern befestigt wurde. Über den Wert dieses Verfahrens läßt sich jedoch noch kein endgültiges Urteil fällen, da es nach Nowakowsky nur einmal zur Anwendung gelangt ist. Für die Überbrückung größerer und größter Defekte von regelloser Gestaltung kommen nur die komplizierten osteoplastischen Methoden in Frage. Von diesen ist das Schimmelbuschsche, Entnahme eines Hautknochenlappens aus dem Brustbein, dem von Photiades und Lardi, die den Lappen aus dem Schlüsselbein wählten, bei weitem überlegen, da erstens die Entnahme des Periostknochenlappens aus der Clavicula technisch recht schwierig ist und es außerdem nachher bereits mehrfach zu einem Schlüsselbeinbruch gekommen ist. Auch das eingeheilte Knochenstück hat zu unangenehmen Beschwerden beim Schlucken geführt, so daß eine Nachresektion notwendig wurde.

Die ausgezeichneten Resultate, die König und Hohmeier im Tierexperiment und auch zum Teil am Menschen mit der 
autoplastischen freien Fascientransplantation bei der Deckung von Blasen, Ösophagus und Darmwanddefekten zu verzeichnen hatten, veranlaßten Hohmeier in einer weiteren Serie von Versuchen den Wert dieses neuen Verfahrens beim Verschluß von Trachealdefekten zu erproben. Die Erfolge waren sehr befriedigend. Bei 7 Hunden, bei denen die freie Fascientransplantation bei einem frischen Trachealdefekt ausgeführt wurde, erfolgte ebenso wie in zwei weiteren Fällen, wo der Defekt erst 8- Io Tage später gedeckt wurde, eine prompte Einheilung des Lappens. Der Lappen wurde siebenmal aus der Fascie des Sternocleido, zweimal aus der des Rectus entnommen. Die Atmung war nach der Operation niemals gestört; schwerere Lungenkomplikationen wurden nicht beobachtet. Hautemphysem trat niemals auf. Bei der Sektion der Tiere zeigte sich die Fascie selbst I2 Wochen nach der Operation fast unverändert, was auch durch die mikroskopische Untersuchung bestätigt wurde. Die Fascie überdeckte ohne Eindellung in guter Spannung den Defekt. Sie war mit dem Perichondrium innig verwachsen; übermäßige Granulationsbildung im Bereich des Implantats wurde nicht gefunden, vielmehr war in einigen Fällen bereits nach 4 Wochen die Fascie von Schleimhaut überzogen.

Beim Menschen ist die autoplastische freie Fascientransplantation zwecks Deckung eines Trachealdefektes bisher nur 2 mal, und zwar mit bestem Erfolge ausgeführt worden. Ein dritter Fall ist von mir im Juni dieses Jahres operiert worden. Im ersten Fall (Levit) handelte es sich um einen Patienten, bei dem im Anschluß an einen Selbstmordversuch eine Trachealfistel zurückgeblieben war und bereits 3 mal vergebliche Versuche mit Lappenplastiken gemacht waren. Ein guldengroßer Lappen wurde aus der Fascia lata femoris nach vorheriger Anfrischung der Wundränder in den Defekt eingesetzt und heilte reizlos ein. Der Erfolg war sehr befriedigend. Die Patientin konnte sofort nach der Operation frei atmen, nach 6 Tagen war die Stimme normal. Bei der 5 Monate p. o. vorgenommenen direkten Tracheoskopie wurde an der Stelle des Implantats eine weißliche, zweihellerstückgroße Fläche gefunden; die übrige Schleimhaut zeigte hellrotes Aussehen. Die Atmung war vollkommen frei. Eine Beweglichkeit der Fascie beim Atmen wurde nicht beobachtet. In dem zweiten 
Fall (Lucas), bei dem auch bereits vorher ein vergeblicher Versuch mit einer Hautlappenplastik gemacht war, wurde der ebenfalls nach Selbstmordversuch entstandene Trachealdefekt vermittelst eines Fascienlappens aus dem Oberschenkel gedeckt. Auch hier erfolgte glatte Einheilung mit gutem funktionellen und kosmetischen Resultat.

In meinem Fall handelte es sich um einen 23 jährigen Musketier N., der sich in selbstmörderischer Absicht am Hals und linken Unterarm mehrere Schnittwunden mit einem anscheinend recht scharfen Rasiermesser beigebracht hatte.

Status praesens (4. VI. I9I3): Mittelgroßer, nur mittelkräftig gebauter Mann, stark anämisch infolge des großen Blutverlustes. Dicht unterhalb des Ringknorpels verlaufen zwei je 9 und $7 \mathrm{~cm}$ lange, in der Mittellinie unter einem. Winkel von ungefähr $45 \mathrm{Grad}$ sich kreuzende Schnittwunden, die die Haut und die seitliche Halsmuskulatur vollkommen durchtrennt haben. Die Muskulatur und Fascie ist stark zerfetzt, der untere Teil des rechten Schilddrüsenlappens durch mehrere Schnitte zerlegt. Die großen Gefäße und Nerven sind unverletzt. Der Ringknorpel und die obersten Trachealringe liegen frei in der Wunde. Der Ringknorpel ist durch mehrere, bis in das Lumen eindringende Schnitte lamellenartig zerteilt. Unterhalb des Ringknorpels sieht man einen klaffenden, ungefähr zehnpfennigstückgroßen Defekt in der vorderen Trachealwand, aus dem sich bei Hustenstößen schaumiges Blut entleert. Atmung frei. Chloroformnarkose. Nachdem die Blutung, die zurzeit nur noch gering war, sorgfältigst gestillt war, wurde durch einige Nähte der Ringknorpel zusammengezogen und der Versuch gemacht, die Trachealwundränder gleichfalls durch Naht miteinander zu vereinigen; die Fäden schnitten jedoch regelmäßig durch. Eine Vereinigung der Wundränder ohne Resektion erschien nicht möglich und wäre auch nur unter Opferung des Ringknorpels möglich gewesen, da die Fäden bei der lamellösen Auflockerung dieses keine Garantie für eine sichere Naht geboten hätten. Ich suchte deshalb den Defekt auf andere Weise zu decken und entnahm hierzu aus der Fascia lata des linken Oberschenkels einen ungefähr $3 \mathrm{qcm}$ großen Fascienlappen. Dieser wurde mit der Muskelseite nach oben in den Defekt gelegt. Die Befestigung des Lappens vermittels Katgutknopfnähten gelang ohne Mühe, nachdem an den vier Ecken vorher Fixationsnähte angebracht waren. Der Lappen überdeckte in guter Spannung, ohne sich bei der Atmung einzuziehen, den Defekt; Luftaustritt erfolgte nicht mehr. Vollkommener WundverschluB, nachdem vorher noch durch einige Katgutnähte die Reste der Muskulatur und Fascie zusammengezogen waren. Versorgung der Unterarmwunde (Sehnennaht). 
Die Wundheilung verlief ohne Störung. Die Atmung war nach der Operation vollkommen frei, der Kranke konnte sofort nachher wieder sprechen und gut schlucken. Bis auf eine kleine Stelle in der Mitte der Naht, wo sich für einige Tage eitriges Sekret entleerte, trat prima intentio ein. Am neunten Tage wurde aus der Wundöffnung ein Katgutfaden ausgestoßen, am nächsten Tage hatte sich eine kleine Trachealfistel gebildet, die jedoch später nach Anlegung einiger Nähte gleichfalls zur Heilung gelangte. Am 29. VII. I9I3 wurde N. dienstfähig zur Truppe entlassen. Entlassungsbefund: Über die Gegend des Ringknorpels ziehen zwei diagonal zueinander verlaufende, 8 und $6 \mathrm{~cm}$ lange reizlose Narben, die an der Kreuzungsstelle sich etwas verbreitern und daselbst mit der Unterlage verwachsen sind. Die Gegend des ehemaligen Trachealdefekts ist durch die Haut deutlich abtastbar. Der Defekt ist fest überbrückt, beim Husten und Pressen sind keine Spannungsänderungen an der Implantationsstelle nachweisbar. Die Atmung ist frei. Die Bewegungen des Kehlkopfes beim Schlucken und Sprechen sind deutlich zu verfolgen; der adhärente Teil der Narbe steigt dabei ausgiebig mit em. por. Die Stimme ist gut tönend, mittellaut, nach längerem Gebrauch hat sie einen etwas unreinen, leicht krächzenden Beiklang. Laryngoskopischer Befund: Die Stimmbänder legen sich bei der Phonation fest aneinander an; die Gegend des Implantats läßt im Spiegelbild bei der Atmung keine Bewegungen erkennen und ist überall von hellroter Schleimhaut überkleidet.

Liegen auch bisher nur die Erfahrungen dreier Fälle vor, bei denen die autoplastische freie Fascientransplantation zwecks Deckung eines Trachealdefektes ausgeführt wurde, so lassen doch diese im Verein mit den äußerst günstigen auch an anderen schleimhauttragenden Organen gewonnenen Resultaten (siehe Sammelreferat Kirschner) die Annahme gerechtfertigt erscheinen, daß diese neue Methode die alten Plastiken an Sicherheit merklich übertrifft. Waren doch z. B. im Fall Levit bereits dreimal und im Fall Lukas einmal vergebliche Versuche mit anderen Plastiken angestellt worden. Von besonderem Wert ist neben der Sicherheit gegenüber den älteren Methoden die Einfachheit der Technik und die guten kosmetischen Resultate.

Bezüglich der Technik noch einige Bemerkungen. Nicht ohne Belang ist die Größe des Fascienlappens. Dieser darf

Anmerkung bei der Korrektur: N. ist dienstfähig geblieben und am 29. IX. I9r3 zur Reserve entlassen worden. Nachuntersuchung am I6. XI. I9I3 hat weitcre Besserung der Stimme ergeben, Atmung frei, Narbe fest und reizlos. $N$. ist vollkommon beschwerdefrei. 
nicht zu klein gewählt werden; er muß mindestens um 3-4 mm die Trachealwundränder überragen und unter straffer Anspannung in eine möglichst exakte Verbindung durch zahlreiche Knopf- bzw. eine fortlaufende Naht mit dem Perichondrium gebracht werden, da dieses, wie die histologischen Untersuchungen ergeben, zunächst das ernährende Element für den Fascienlappen darstellt. Der Fascienlappen ist möglichst schonend zu behandeln und sofort nach Entnahme in den Defekt einzusetzen. Als Nahtmaterial ist am meisten dünnstes Katgut (Kuhn) zu empfehlen. Leider stand mir letzteres nicht zur Verfügung und glaube ich, auch in meinem Fall auf das zu dick gewählte Nahtmaterial die nach to Tagen entstandene Trachealfistel zurückführen zu müssen. Eine Drainage oder Tamponade der Wunde ist auf jeden Fall zu unterlassen, da hierdurch die Ernährung der Fascie in Frage gestellt wird. Die Gefahr einer Infektion der Wunde ist bezüglich der Einheilung des Lappens nicht so hoch zu veranschlagen, da die Fascie bei ihrer ausgesprochenen Widerstandsfähigkeit selbst stärkere Infektionen mit Glück überwindet. Als Entnahmestelle für den Fascienlappen eignet sich am meisten der Oberschenkel, und zwar der straffe Tractus ilio-tibialis, da dieser eine wesentlich größere Widerstandsfähigkeit wie die Halsfascie besitzt und außerdem auf diese Weise entstellende Narben in der Halsgegend vermieden werden. Die Befürchtung, daß sich etwa infolge einer postoperativen Muskelhernie Beschwerden einstellen könnten, ist, wie die Nachuntersuchungen bei zahlreichen anderen Fascienplastiken gezeigt haben, vollkommen unbegründet.

Zum Schluß noch einige Worte über die Behandlung frischer Trachealwunden. In erster Linie ist stets für eine exakte Blutstillung und die Abwendung der unmittelbaren Asphyxiegefahr Sorge zu tragen. Bezüglich der weiteren Behandlung galt im allgemeinen früher der Grundsatz, die offene Wundbehandlung durchzuführen, da man sich von der primären Naht keinen Erfolg versprach und sie wegen Begünstigung von Infektion, Hautemphysem und Larynxödem direkt für gefährlich erachtete. Nur bei stark klaffenden Wunden der ganz oder fast ganz durchtrennten Luftröhre wurde der Versuch einer Vereinigung der Seitenwinkel der Haut und der Trachealwinkel für berechtigt 
angesehen. Die Gefahren der primären Naht sind früher entschieden wesentlich überschätzt und die Vorteile sind so wesentlich, daß die Mehrzahl der Chirurgen heutigen Tages für diese mit oder ohne prophylaktische Tracheotomie eintritt. Das subjektive Befinden der Kranken wird durch die Naht wesentlich gehoben, da der quälende Hustenreiz fortfällt und sie sofort nach der Operation sprechen und schlucken können. Die Behandlungsdauer wird bedeutend verkürzt, die Gefahr der Entstehung einer Trachealfistel und einer sich daran anschließenden Stenose erheblich herabgesetzt. Die prophylaktische Tracheotomie, die von vielen Chirurgen immer noch ausgeführt wird, kann man meines Erachtens bei den frischen Trachealverletzungen (nicht bei den Larynxverletzungen) bei stationärer Behandlung in den meisten Fällen entbehren. Nur unstillbare, profuse Schleimhautblutungen, die eine vorübergehende Tamponade erheischen und Rekurrensverletzungen rechtfertigen diesen Eingriff. Bei den Trachealdefekten, die sich nicht durch die Naht verschließen lassen, ist die primäre Plastik, und zwar, wenn irgend möglich, die autoplastische freie Fascientransplantation auszuführen, da diese am sichersten einen festen Wundverschluß garantiert und daher am ehesten Hautemphysem und Infektion vorbeugt.

\section{Literaturverzeichnis.}

I. B arracz, Ein Beitrag zur Trachealplastik. Wiener klin. Wochenschrift I90I, Nr. 49, S. 1054 .

2. Di effenbach, Dic operative Chirurgie. Leipzig I845.

3. GroBe, Trachealknorpeldefekte mit Silberdrahtnetzbedeckung. Zentralblatt f. Chir. I90 I, Nr. 45, S. I Iro.

4. Hohmeier, Experimente über Verschluß von Wunden und Überbrückung von Defekten schleimhauttragender Körperkanäle und thöhlen durch freie Autoplastik. Verhandlungen der deutschen Gesellschaft für Chirurgie I9r I, S. II I.

5. Kirschner, Die praktischen Ergebnisse der freien Fascientransplantation. Verhandlungen der deutschen Gesellschaft für Chirurgie I910, S. 223.

6. Derselbe, Der gegenwärtige Stand und die nächsten Aussichten der autoplastischen freien Fascienübertragung. Beitr. z. klin. Chir. 1913, Bd. 86, S. 5 . 
7. König, Die Deckung von Defekten der vorderen Trachealwand. Berliner Klinik I896, Nr. 5r, S. I I 29.

8. Derselbe, Neue Wege der plastischen Chirurgie (Verlötung und Überbrückung). Verhandlungen der deutschen Gesellschaft für Chirurgie I9II, S. 92.

9. $\mathrm{K} \ddot{\mathrm{u} s t e r}$, Zur Deckung von Trachealdefekten. Verhandlungen der deutschen Gesellschaft für Chirurgie I893, S. 80.

Io. L evit, Deckung von Trachealdefekten durch freie Plastik aus der Fascia lata femoris. Arch. f. klin. Chir. I912, Bd. 97, S. 686.

II. Lukas, Über freie Plastik der Fascia lata. Arch. f. klin. Chir. I9I3, Bd. I00, S. I 29.

I2. Mangold, v., Die Einpflanzung von Rippenknorpel in den Kehlkopf zur Heilung schwerer Stenosen und Defekte. Verhandlungen der deutschen Gesellschaft für Chirurgie 1900, S. 460.

13. Nowakowsky, Beitrag zur Trachealplastik. Arch. f. klin. Chir. I909, Bd. 90, S. 847 .

14. Photiades u. La rdi, Contributions à la chirurgie des voies réspiratoires. Revue méd. de la Suisse, Rom 1893.

15. Schimmelbusch, Zur Deckung von Trachealdefekten. Verhand. lungen der deutschen Gesellschaft für Chirurgie I893, S. 78.

16. Velpeau, J. F. Malgaine, Léon le Fort, Manuel médecine opératoire. Paris 1874, Bd. I, S. 507. 